\title{
Salicylic acid as a peeling agent: a comprehensive review
}

\section{Tasleem Arif \\ Postgraduate Department of Dermatology, STD and Leprosy, Government Medical College, Srinagar, Jammu and Kashmir, India}

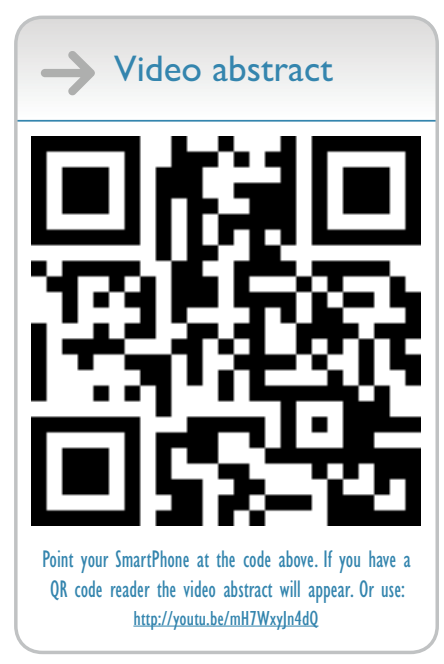

Correspondence: Tasleem Arif Postgraduate Department of Dermatology, STD and Leprosy, Government Medical College, Srinagar, Jammu and Kashmir 1900II, India Tel +9l 9557480345

Email dr_tasleem_arif@yahoo.com
This article was published in the following Dove Press journal:

Clinical, Cosmetic and Investigational Dermatology

26 August 2015

Number of times this article has been viewed

Abstract: Salicylic acid has been used to treat various skin disorders for more than 2,000 years. The ability of salicylic acid to exfoliate the stratum corneum makes it a good agent for peeling. In particular, the comedolytic property of salicylic acid makes it a useful peeling agent for patients with acne. Once considered as a keratolytic agent, the role of salicylic acid as a desmolytic agent, because of its ability to disrupt cellular junctions rather than breaking or lysing intercellular keratin filaments, is now recognized and is discussed here. Salicylic acid as a peeling agent has a number of indications, including acne vulgaris, melasma, photodamage, freckles, and lentigines. The efficacy and safety of salicylic acid peeling in Fitzpatrick skin types I-III as well as in skin types V and VI have been well documented in the literature. This paper reviews the available data and literature on salicylic acid as a peeling agent and its possible indications. Its properties, efficacy and safety, the peeling procedure, and possible side effects are discussed in detail. An account of salicylism is also included.

Keywords: acne vulgaris, desmolytic agent, melasma, photodamage, salicylic acid

\section{Introduction}

Chemical peeling is a safe, efficacious, and cost-effective procedure for treating various skin disorders and for enhancing cosmetic appearance. The principle of peeling involves controlled chemical injury to the skin in order to prompt it to rejuvenate, leading to smoothening of the skin and improvement of its surface texture. ${ }^{1}$ Chemical peels can be classified in different ways. A useful approach is to classify them according to the level of injury caused to the skin, which determines the indications they can be used to treat. Accordingly, chemical peels can be divided into three broad categories, ie, superficial, medium-depth, and deep. Superficial peels cause injury to the epidermis, so are used to treat superficial conditions, including melasma, acne, and dyschromias. Medium-depth peels penetrate to the papillary dermis, and are useful in the treatment of solar keratoses, dyschromias, and pigment disorders. Deep peels cause necrosis to the level of the reticular dermis, so are indicated for deep wrinkles, severe photoaging, and deep scars. ${ }^{1}$ Salicylic acid (SA) is a member of a group of compounds known as hydroxy acids, which are widely used for a number of cosmetic indications because of their many important properties. ${ }^{2}$ The aim of this review is to give a detailed account of SA as a peeling agent in cosmetic dermatology. This review also resolves some queries regarding the position of SA among hydroxy acids; its mechanism of action being desmolytic rather than true keratolytic, and its safety among dark skinned people. submit your manuscript | www.dovepress.com
Dovepress 


\section{Definition of chemical peeling}

Chemical peeling is the process of causing controlled chemical injury to the skin (partial or complete epidermis with or without dermis) by application of a chemical peeling agent that causes exfoliation of the superficial layers of the skin, leading to removal of superficial lesions followed by regeneration of new epidermal and dermal tissues. ${ }^{3}$

\section{Historical background}

SA has been used topically to treat various skin disorders for more than 2,000 years. ${ }^{4}$ In the first century AD, Pliny used willow bark (as a source of SA) to treat calluses and corns. Buchner, Brugnatelle, and Fontana isolated salicin from willow bark in the late 1820s, which was later refined by Leroux. The ability of SA to soften and exfoliate the stratum corneum was discovered in the 1860s. ${ }^{5}$ Paul Gerson Unna, a German dermatologist, is credited with describing the properties and use of SA. Being a comedolytic agent, SA is used in many topical acne preparations. It is also known to enhance the penetration of other topical agents. ${ }^{6}$

\section{Chemistry and properties}

Chemically, SA is 2-hydroxybenzoic acid or orthohydrobenzoic acid. Sources of SA and salicylates include willow bark, sweet birch, and wintergreen leaves. However, SA can also be synthesized artificially., ${ }^{4}$

Kligman $^{8}$ described SA as a $\beta$-hydroxy acid, but Yu and Van Scott $^{9}$ have classified it as a phenolic aromatic acid. SA has carboxyl (-COOH) and hydroxyl (-OH) groups directly attached to an aromatic benzene ring, unlike a true $\beta$-hydroxy acid, which contains an aliphatic carbon atom chain. Secondly, the hydroxyl group of SA has acidic properties whereas the hydroxyl group of a true $\beta$-hydroxy acid is neutral. ${ }^{89}$ Moreover, the carbon atoms of aromatic compounds are counted in arabic numerals (eg, 1, 2, 3) rather than the Greek letter designations (eg, alpha, beta, gamma) applying to aliphatic structures (non-aromatic compounds). It is possible that SA was labeled as a $\beta$-hydroxy acid at a time when $\beta$-hydroxy acid peels were introduced in the market in order to exploit the benefit of the popularity of $\alpha$-hydroxy acids. ${ }^{10}$

SA is a lipid-soluble agent, in contrast with the $\alpha$-hydroxy acids (such as glycolic acid), and is therefore miscible with epidermal lipids and sebaceous gland lipids in hair follicles. ${ }^{7}$ SA has keratolytic and comedolytic properties, although the exact mechanisms involved are not clear. ${ }^{11} \mathrm{SA}$ also decreases secretion of sebum in patients with acne, which adds to its therapeutic effect in these patients. Marczyk et al studied the effect of $50 \%$ pyruvic acid and $30 \%$ SA peels on skin lipid film in patients with acne vulgaris, and concluded that SA had a greater sebumetric effect than pyruvic acid. ${ }^{12}$ SA decreases adhesion of corneocytes, and causes loosening of these cells and their subsequent detachment. ${ }^{13-15}$ Being a lipophilic agent, SA removes intercellular lipids, which are linked covalently to the cornified envelope surrounding the surface epithelial cells. ${ }^{16}$ This antihyperplastic effect of SA on the epidermis has been used by many dermatologists in chemical peeling of skin. ${ }^{17,18}$

Cohesion of epidermal cells in the skin depends upon desmosomes, which contain many proteins, including desmogleins. It has been found that SA, being an organic acid, extracts desmosomal proteins including desmogleins. As a result of this action, the cohesion of epidermal cells is lost, leading to exfoliation. ${ }^{19}$ Thus, SA should now be regarded as a desmolytic agent rather than as a keratolytic agent in so far as its mechanism of action is concerned, because it works by disrupting cellular junctions rather than breaking or lysing intercellular keratin filaments. ${ }^{20}$ Imayama et al studied histological changes in the skin of hairless mice following peeling with SA, and reported loss of cornified cells followed by activation of epidermal basal cells and underlying fibroblasts. They concluded that peeling with SA can lead to alterations in the underlying dermal tissue without directly wounding the skin. ${ }^{19}$ SA does not affect mitotic activity in human epidermal cells. ${ }^{13}$ However, Weirich et al studied the antihyperplastic effect of $\mathrm{SA}$ in the guinea pig epidermis and concluded that there is a reduction in hyperplasia of viable epidermal cells. ${ }^{21}$ Use of SA on human skin causes thinning of the corneal layer without any change in the thickness of the epidermis. ${ }^{22}$

Aspirin, chemically known as acetylsalicylic acid, is a well-known analgesic and antipyretic substance causing irreversible inhibition of prostaglandin synthesis. Being a salicylate, SA also has anti-inflammatory properties. The concentration at which the anti-inflammatory action of SA is most pronounced is between $0.5 \%$ and $5 \%(\mathrm{w} / \mathrm{w}) .^{11,23,24}$

Salicylates and their derivatives have been used in sunscreen formulations. The aromatic benzene ring in the salicylate converts ultraviolet radiation into longer wave radiation that is felt as heat, which explains the mechanism of action in sunscreen preparations. ${ }^{25} \mathrm{SA}$ also has antimicrobial and antifungal properties. Whitfield's ointment, which contains benzoic acid and SA, has been used for its antifungal properties. ${ }^{26} \mathrm{SA}$ also has an anesthetic effect, which is seen during the peeling procedure and increases the patient's ability to tolerate the procedure. ${ }^{6}$ 


\section{Concentrations and formulations}

Various preparations of SA have been used for peeling purposes. Aronsohn and Swinehart have used 50\% SA ointment, ${ }^{17,18}$ and the formulation they used for actinic damage and pigmentary changes consisted of SA 50\%, 16 drops of methyl salicylate, and $112 \mathrm{~g}$ of Aquaphor. ${ }^{17} \mathrm{SA}$ has also been used in ethyl alcohol solutions containing SA 10\%, 20\%, $30 \%, 40 \%$, and $50 \%(\mathrm{w} / \mathrm{v}) .{ }^{27}$ Newer formulations of SA have been introduced. A formulation of SA $30 \%$ in polyethylene glycol vehicle has been shown to be less absorbed through the intact skin of hairless mice. The risk of salicylism is low in humans with intact skin during chemical peeling using this formulation. ${ }^{28}$ A study by Dainichi et al showed that chemical peeling with SA in polyethylene glycol helps to reduce as well as prevent ultraviolet B-induced skin tumors in hairless mice. ${ }^{29}$ Hence, this formulation holds potential for use in chemical peeling due to its added safety and anticarcinogenic properties. The properties and indications of SA are dependent on the concentration used. The various concentrations of SA that have been used in dermatology and cosmetic procedures are listed in Table 1.6,17,30-33

\section{Indications}

SA as a peeling agent has been studied by various dermatologists. Aronsohn used 50\% SA ointment with excellent results in 81 patients with pigmentation, freckles, and photoaging of the hands. ${ }^{18}$ Swinehart used a $50 \%$ SA ointment paste containing buffered methyl salicylate and croton oil for the treatment of actinically damaged skin, lentigines, and pigmented keratosis on the forearms and dorsal aspect of the hands, and reported excellent results. ${ }^{17}$ Being a lipophilic agent and having an ability to concentrate in the pilosebaceous apparatus, SA peels are a good therapeutic option for comedonal acne, and can be a good adjunctive modality for treating open and closed comedones, post-acne erythema, and hyperpigmentation. ${ }^{34}$ The efficacy of SA in the treatment of photoaging and acne has been described in patients with Fitzpatrick skin types I-III as well as in skin types V and VI. ${ }^{35}$

Table I Various concentrations of SA used in dermatology and cosmetics

\begin{tabular}{ll}
\hline SA concentration & Uses \\
\hline $0.5 \%-10 \%^{30,31}$ & Acne \\
$3 \%-6 \%^{32}$ & Hyperkeratotic disorders like psoriasis, \\
& ichthyoses, keratosis pilaris \\
$5 \%-40 \%{ }^{33}$ & Warts, corns \\
$50 \%{ }^{17}$ & Actinic damage and pigmented lesions \\
$20 \%-30 \%^{6}$ & Superficial chemical peeling of face \\
\hline
\end{tabular}

Abbreviation: SA, salicylic acid.
Kligman and Kligman used SA as a superficial peeling agent in 50 women with mild to moderate photodamage, and reported improvement in surface roughness and pigmented lesions, along with a reduction in fine lines. ${ }^{36}$ Grimes treated 25 patients from a darker racial ethnic group who had acne vulgaris, melasma, or post-inflammatory hyperpigmentation with $20 \%$ and $30 \%$ SA peels, and reported good efficacy with minimal side effects. ${ }^{6}$ The various indications for SA peeling are listed in Figure 1.

\section{Contraindications}

SA peels have been found to be safe and well tolerated by all racial/ethnic groups and in all skin types (Fitzpatrick I-VI). The possible contraindications to SA peeling are mentioned in Figure 2.

\section{Physician's qualifications}

This is an important consideration before undertaking peeling to avoid the hazards of an imperfect peel, which can simply damage the patient's face. There are certain prerequisites that should be met by physicians before embarking on chemical peels in their patients, the more important of which are described below. ${ }^{3}$

The treating physician should be a trained dermatologist who has acquired adequate training in chemical peeling during post-graduate training in dermatology. In India, most medical colleges do not teach esthetics and cosmetics in the post-graduate years. However, such training can be acquired later at a center that routinely provides education and training in cosmetic and cutaneous surgery. Focused workshops can also provide such training. The physician should be well versed in the basic chemistry of peels, and should have a good understanding of acids, bases, and the $\mathrm{pH}$ and $\mathrm{pK}_{\mathrm{a}}$ of peeling agents. A thorough knowledge of the mechanisms and properties of the peeling agent used is of utmost importance for a good therapeutic outcome. The concept of wound healing

\begin{tabular}{|l|}
\hline - Acne vulgaris \\
\hline - Melasma \\
\hline - Post-inflammatory hyperpigmentation \\
\hline - Freckles \\
\hline - Lentigines \\
\hline - Photodamage (mild to moderate) \\
\hline - Texturally roughened skin \\
\hline - Fine wrinkling, Glogau I \\
\hline
\end{tabular}

Figure I Indications for salicylic acid peeling 


\begin{tabular}{|c|}
\hline - Contact allergy to salicylates \\
\hline $\begin{array}{l}\text { - Active dermatitis at the peeling } \\
\text { site }\end{array}$ \\
\hline - Active infection \\
\hline - Acute viral infection \\
\hline - Pregnancy \\
\hline - Active dermatitis at the site \\
\hline $\begin{array}{l}\text { - Use of isotretinoin during the } \\
\text { 3- to 6-month peeling procedure }\end{array}$ \\
\hline - Skin malignancy \\
\hline - Tanned skin \\
\hline
\end{tabular}

Figure 2 Contraindications to salicylic acid peeling.

after a controlled chemical skin injury should be clear to the physician, who should also have a thorough knowledge of the pathogenesis and therapeutic aspects of the diseases being treated, such as melasma and acne. Finally, the physician should have a mastery of all aspects of potential postoperative complications, including preventing, recognizing, and managing them.

\section{Pre-peel preparation}

Appropriate patient selection and assessment of each individual's skin condition is mandatory before considering a chemical peel. The treating dermatologist must evaluate the patient for the possible indications, look for any contraindications, discuss the procedure in detail, and assess the patient's expectations and anticipation of results. The potential risks of the procedure as well as the limitations must be discussed. The patient's skin type should be evaluated using Fitzpatrick's classification. ${ }^{37}$ The level of photodamage should also be assessed using the Glogau classification. Prior to the chemical peel, a detailed history and cutaneous examination must be performed in all patients. Areas to be peeled should be photographed, and include full-face frontal and lateral views. An informed consent must be signed prior to performing the peeling procedure.

Several pre-peel regimens have been used by dermatologists. Various combinations involving topical tretinoin, $\alpha$-hydroxy acids, hydroquinone, kojic acid, and low potency steroids have been used to prime the skin prior to the peel. Patients should be advised to avoid smoking, minimize their exposure to sunlight, and apply broad-spectrum sunscreens. ${ }^{3}$ Pre-peel regimens differ between acne vulgaris, photodamage, and hyperpigmentation, including melasma and post-inflammatory hyperpigmentation. ${ }^{3}$

Topical retinoids (tretinoin, tazarotene, retinol formulations) applied for 2-6 weeks prior to peeling thin the stratum corneum and enhance epidermal cell turnover. Such agents also reduce the content of epidermal melanin and expedite epidermal healing. Retinoids also enhance the penetration of the peeling agent. They should be discontinued several days prior to the peeling procedure. Retinoids can be resumed postoperatively after all evidence of peeling and irritation subsides. In contrast with photodamage, when treating conditions such as melasma, post-inflammatory hyperpigmentation, and acne, as well as darker skin types, retinoids should be discontinued 1 or 2 weeks before peeling or even eliminated from the pre-peel regimen to avoid post-peel complications, such as excessive erythema, desquamation, and post-inflammatory hyperpigmentation. ${ }^{6}$

Topical hydroquinone has been used as a priming agent for patients affected with pigment dyschromias. In this regimen, $4 \%$ hydroquinone is applied twice daily for 2-4 weeks prior to the peel and is resumed 2 days post-peel. The combination of pre-peel application of $4 \%$ hydroquinone twice daily with peeling produces substantial decreases in the intensity of hyperpigmentation in both post-inflammatory hyperpigmentation and melasma. ${ }^{38}$ It should be noted that prolonged use of high concentrations of hydroquinone $(6 \%-10 \%)$ may lead to exogenous ochronosis. Topical $\alpha$-hydroxy acid or polyhydroxy acid formulations have also been used in prepeel regimens but are less aggressive in terms of impacting peel outcomes.

Topical and systemic therapies may be initiated $2-4$ weeks prior to the peeling procedure in patients with acne vulgaris. Topical benzoyl peroxide and other topical antibiotic formulations can be used daily and discontinued 1 or 2 days prior to peeling. Broad-spectrum sunscreen formulations (ultraviolet A and ultraviolet B) should be applied frequently. ${ }^{6}$ On the day of the chemical peel, it is helpful to advise patients to start antiviral prophylaxis and continue it for 7-10 days; some dermatologists prefer 2 days before the peel and treat for a total duration of $7-10$ days. ${ }^{39}$

\section{Peeling procedure}

The standard procedure for chemical peeling with SA involves use of a $20 \%$ or $30 \%$ SA formulation in an ethanol base. The peels are repeated at an interval of 2-4 weeks. Peak results are visible after a series of 3-6 chemical peels depending on the severity of the condition being treated and skin type. It is 
wise to perform the initial peel with a $20 \%$ SA formulation in order to assess sensitivity and reactivity of the patient's skin to the peeling agent. Before starting the peel, the face should be thoroughly cleansed with alcohol and/or acetone to remove oils from the skin. The peel is then applied. Various methods can be used to perform the peel. Usually, $2 \times 2$ wedge sponges, cotton-tipped applicators, and $2 \times 2$ gauze sponges are used for application. In clinical practice, $2-3$ coats of SA are usually applied in one sitting. The manner in which the peel is applied on the face can vary. One good method is to apply the peel first to the medial cheeks and working laterally, followed by the perioral area, then chin, and lastly the forehead. The peel is then left on for 3-5 minutes. During the procedure, most patients complain of mild burning and a stinging sensation. After 1-3 minutes, some patients may experience anesthesia of the face due to the anesthetic properties of the SA peel. The sensation of burning and stinging can be reduced using a portable handheld fan or ice packs. Within 30 seconds to 1 minute of peeling, a white precipitate is formed, as a result of crystallization of the SA. It must be remembered that the frost seen in a SA peel represents precipitated SA, while the frost in a trichloroacetic acid peel is formed by precipitation of skin proteins. ${ }^{3}$ Once frosting has occurred, it means that the patient will observe some crusting and peeling after the procedure. This crusting and peeling may be quite acceptable for treating a patient with photodamage, but when treating melasma and other pigment dyschromias, minimal or no frosting is the preferred option. The SA peel has an advantage over the $\alpha$-hydroxy acid peel in that the former does not need to be neutralized and the frost is visible once the peel is complete. ${ }^{10}$ Once the peel has had sufficient contact time, which varies in the range of 3-5 minutes, the face is rinsed thoroughly with tap water. A bland cleanser can be used to remove any residual SA precipitate. After rinsing, a bland moisturizer is applied to the skin.

\section{Post-peeling care}

For the first 48 hours post-peel, bland moisturizers and cleansers are continued or until all post-peel irritation has ceased. Patient can then resume their topical skin care formulations, which may include topical demelanizing agents, topical anti-acne medications, and/or retinoids. Excessive desquamation and irritation in the post-peel period can be treated with low to high potency topical steroids. It should be noted that topical steroids have been found to be effective in mitigating post-peel inflammation and reducing post-inflammatory hyperpigmentation. However, any hyperpigmentation remaining after the peeling procedure responds to topical hydroquinone and frequent use of sunscreens.

\section{Side effects}

Peeling with SA has been well documented in the literature, but certain adverse effects (Table 2 ) occur, which are usually mild and transient. Lee and Kim performed SA peeling in 35 Korean patients with acne vulgaris and found prolonged erythema that lasted more than 2 days in $8.8 \%$ of their patients. ${ }^{40}$ Dryness has been reported in $32.3 \%$ of cases. This can be managed with frequent application of topical moisturizers. Grimes reported intense exfoliation and crusting following SA peeling in $17.6 \%$ and $11.7 \%$, respectively, in her patients. However, exfoliation cleared in 7-10 days. There were no cases of scarring or persistent post-inflammatory dyschromias. ${ }^{6}$ In a study that investigated the safety and efficacy of SA chemical peels in darker ethnic groups (20 African Americans and five Hispanics), 16\% experienced mild side effects. Temporary crusting and hypopigmentation was seen in one patient, which cleared in 7 days. Transient dryness and hyperpigmentation was seen in three patients, but resolved in 7-14 days. The author concluded that a SA peel is safer in individuals of darker ethnicity. ${ }^{38}$

\section{Salicylism}

SA is absorbed readily when applied topically to the skin, ${ }^{25}$ and can be detected in urine within 24 hours applied to skin with erythroderma. ${ }^{41}$ The absorption of SA can be increased topically when it is combined with a hydrophilic base or kept under occlusion. ${ }^{42,43}$ Systemic toxicity due to cutaneous absorption is a very rare phenomenon, but is a serious concern. In high concentrations, salicylates are toxic to the central nervous system. The clinical presentation of SA toxicity includes nausea, vomiting, dizziness, psychosis, stupor, and consequently coma and death. ${ }^{23,44}$ Increased labyrinthine pressure can be the cause for associated tinnitus. Stimulation of the respiratory center in the medulla can occur, and manifests as marked hyperventilation leading to respiratory

Table 2 Side effects of salicylic acid peeling

- Prolonged erythema

- Intense exfoliation

- Crusting

- Dryness

- Pigmentary dyschromias

- Systemic toxicity, salicylism

- Hypoglycemia

- Contact sensitization 
alkalosis. However, in infants and children, metabolic acidosis can also occur. ${ }^{23}$ It has been seen that salicylate toxicity can occur when the concentration in the blood exceeds $35 \mathrm{mg} / \mathrm{dL}$, which can rarely occur during the peeling procedure. ${ }^{25}$ In dermatological practice, salicylate toxicity been reported when $20 \%$ SA is applied to $50 \%$ of the body surface area. This has been mentioned with the use of $40 \%$ and $50 \%$ SA paste preparations ${ }^{17}$ However, Fung et al studied the relative bioavailability of SA following dermal application of a $30 \%$ SA skin peel preparation, and concluded that facial peeling using the $30 \%$ formulation should not pose any significant risks to the systemic health in patients. ${ }^{45}$

\section{Hypoglycemia}

Systemic absorption of salicylates can affect glucose metabolism and its utilization in cells. This can lead to hypoglycemia, which is mostly seen in patients with uremia. These patients have reduced protein binding of salicylates as a result of which the free form in the blood increases relatively. ${ }^{46}$

\section{Contact allergy}

SA is a weak contact sensitizer. ${ }^{47}$ However, only a few cases have been recorded. ${ }^{4}$ Patients who give a history of allergic contact dermatitis on exposure to SA preparations may not be allergic to SA, but may be allergic to other components of the preparation. Lachapelle and Leroy reported on two patients who had an allergic contact dermatitis to a SA wart formulation and negative patch test results for SA, but had a positive patch test result for colophony which was present in the wart preparation. ${ }^{48}$

\section{SA peeling and pregnancy}

SA is classified by the US Food and Drug Administration as a pregnancy category $\mathrm{C}$ drug. ${ }^{33}$ Use of SA peels is not recommended during pregnancy because the structure of SA is closely related to that of aspirin. Use of aspirin in pregnancy has been associated with miscarriage, birth defects, bleeding complications, and salicylism. On the other hand, $\alpha$-hydroxy acid peels are labeled as category B and can be used safely in pregnancy. ${ }^{49}$

\section{Conclusion}

$\mathrm{SA}$ is a safe and efficacious peeling agent for a number of dermatological and cosmetic problems, including acne vulgaris, melasma, photodamage, freckles, and lentigines. It can be safely used in dark skin types. Newer formulations of SA containing polyethylene glycol have shown promising results in decreasing and preventing skin tumors in mice, making it a potentially attractive peeling agent for use in the future.

\section{Disclosure}

The author reports no conflicts of interest in this work.

\section{References}

1. Rendon Mi, Berson DS, Cohen JI, Roberts WE, Starker I, Wang B. Evidence and considerations in the application of chemical peels in skin disorders and aesthetic resurfacing. J Clin Aesthet Dermatol. 2010;3: $32-43$.

2. Kornhauser A, Coelho SG, Hearing VJ. Applications of hydroxy acids: classification, mechanisms, and photoactivity. Clin Cosmet Investig Dermatol. 2010;3:135-142.

3. Khunger N. Standard guidelines of care for chemical peels. Indian $J$ Dermatol Venereol Leprol. 2008;74:S5-S12.

4. Lin AN, Nakatsui T. Salicylic acid revisited. Int J Dermatol. 1998;37: 335-342.

5. Draelos ZD. Rediscovering the cutaneous benefits of salicylic acid. Cosm Derm. 1997;10 Suppl 4:4.

6. Grimes PE. Salicylic acid. In: Tosti A, Grimes PE, Padova MP, editors. Color Atlas of Chemical Peels. 2nd ed. New York, NY, USA: SpringerVerlag; 2006.

7. Brackett W. The chemistry of salicylic acid. Cosmet Derm. 1997; 10 Suppl 4:5-6.

8. Kligman AM. Salicylic acid: an alternative to alpha-hydroxy acids. J Geriatr Dermatol. 1997;5:128-131.

9. Yu RJ, Van Scott EJ. Salicylic acid: not a beta-hydroxy acid. Cosmet Derm. 1997;10:27.

10. Baumann L, Saghari S. Chemical peels. In: Baumann L, Saghari S, Weisberg E, editors. Cosmetic Dermatology: Principles and Practice. 2nd ed. New York, NY, USA: McGraw-Hill Companies; 2009.

11. Draelos ZD. Salicylic acid in the dermatologic armamentarium. Cosmet Derm. 1997;10 Suppl 4:7-8.

12. Marczyk B, Mucha P, Budzisz E, Rotsztejn H. Comparative study of the effect of $50 \%$ pyruvic and $30 \%$ salicylic peels on the skin lipid film in patients with acne vulgaris. J Cosmet Dermatol. 2014;13:15-21.

13. Roberts DL, Marshall R, Marks R. Detection of the action of salicylic acid on the normal stratum corneum. Br J Dermatol. 1980;102:191-196.

14. Marks R, Davies M, Cattell A. An explanation for the keratolytic effect of salicylic acid. J Invest Dermatol. 1975;64:283.

15. Davies M, Marks RL. Studies on the effect of salicylic acid on normal skin. Br J Dermatol. 1976;95:187-192.

16. Lazo ND, Meine JG, Downing DT. Lipids are covalently attached to rigid corneocyte protein envelope existing predominantly as beta-sheets: a solid state nuclear magnetic resonance study. J Invest Dermatol. 1995; 105:296-300.

17. Swinehart JM. Salicylic acid ointment peeling of the hands and forearms. Effective nonsurgical removal of pigmented lesions and actinic damage. J Dermatol Surg Oncol. 1992;18:495-498.

18. Aronsohn RB. Hand chemosurgery. Am J Cosmet Surg. 1984;1: 24-28.

19. Imayama S, Ueda S, Isoda M. Histologic changes in the skin of hairless mice following peeling with salicylic acid. Arch Dermatol. 2000;136: 1390-1395.

20. Del Rosso J. Pharmacotherapy update: current therapies and research for common dermatologic conditions. The many roles of topical salicylic acid. Skin Aging. 2005;13:38-42.

21. Weirich EG, Longauer JK, Kirkwood AH. Dermatopharmacology of salicylic acid. II. Epidermal anti-hyperplastic effect of salicylic acid in animals. Dermatologica. 1975;151:321-332.

22. Loden M, Bostrom P, Kneczke M. Distribution and keratolytic effect of salicylic acid and urea in human skin. Skin Pharmacol. 1995;8: $173-178$. 
23. Burke A, Smyth E, Fitzgerald Garret A. Analgesic antipyretic and anti-inflammatory agents. In: Brunton LL, Lazo JS, Parker K, editors. Goodman and Gilman's The Pharmacological Basis of Therapeutics. 11th ed. New York, NY, USA: McGraw-Hill; 2006.

24. Weirich EG, Longauer JK, Kirkwood AH. Dermatopharmacology of salicylic acid. III. Topical contra-inflammatory effect of salicylic acid and other drugs in animal experiments. Dermatologica. 1976; 152:87-99.

25. Adam BH, Julio CC, Dana MK, Andrew NL. Agents used for treatment of hyperkeratosis. In: Wolverton SE, editor. Comprehensive Dermatologic Drug Therapy. 3rd ed. New York, NY, USA: Elsevier Saunders; 2013.

26. Wright S, Robertson VJ. An institutional survey of tinea capitis in Harare, Zimbabwe and a trial of miconazole cream versus Whitfield's ointment in its treatment. Clin Exp Dermatol. 1986;11:371-377.

27. Draelos ZD. Atlas of Cosmetic Dermatology. New York, NY, USA: Churchill Livingstone; 2000.

28. Ueda S, Mitsugi K, Ichige K, et al. New formulation of chemical peeling agent: $30 \%$ salicylic acid in polyethylene glycol. Absorption and distribution of 14C-salicylic acid in polyethylene glycol applied topically to skin of hairless mice. J Dermatol Sci. 2002;28:211-218.

29. Dainichi T, Ueda S, Isoda M, et al. Chemical peeling with salicylic acid in polyethylene glycol vehicle suppresses skin tumour development in hairless mice. Br J Dermatol. 2003;148:906-912.

30. Hjorth N. Traditional topical treatment of acne. Acta Derm Venereol Suppl (Stockh). 1980;Suppl 89:53-56.

31. Olsen TG. Therapy of acne. Med Clin North Am. 1982;66:851-871.

32. Olin B. Facts and Comparisons. St Louis, MO, USA; JB Lippincott Co; 1990.

33. Bowe WP, Shalita AR. Effective over-the-counter acne treatments. Semin Cutan Med Surg. 2008;27:170-176.

34. Monheit GD, Chastian MA. Chemical and mechanical skin resurfacing. In: Bolognia JL, Jorizzo JL, Rapini RP, editors. Dermatology Philadelphia, PA, USA: Mosby; 2003.

35. Bourelly PE, Lotsikas-Baggili AJ. Chemexfoliation and superficial skin resurfacing. In: Burgess CM, editor. Cosmetic Dermatology. 1st ed. Heidelberg, Germany: Springer-Verlag; 2005.
36. Kligman D, Kligman AM. Salicylic acid peels for the treatment of photoaging. Dermatol Surg. 1998;24:325-328.

37. Skin Inc. The Fitzpatrick Skin Type Classification Scale. November, 2007. Available from: http://www.skininc.com/skinscience/physiology/10764816.html. Accessed January 7, 2014.

38. Grimes PE. The safety and efficacy of salicylic acid chemical peels in darker racial-ethnic groups. Dermatol Surg. 1999;25:18-22.

39. Mendelsohn JE. Update on chemical peels. Otolaryngol Clin North Am. 2002;35:55-72.

40. Lee HS, Kim IH. Salicylic acid peels for the treatment of acne vulgaris in Asian patients. Dermatol Surg. 2003;29:1196-1199.

41. Kvorning SA. On ointments containing salicylic acid. Acta Dermatol Venereol. 1954;34:89-91.

42. Stolar ME, Rossi GV, Barr M. The effect of various ointment bases on the percutaneous absorption of salicylates. I. Effect of type of ointment base. J Am Pharm Assoc. 1960;49:144-147.

43. Taylor JR, Halprin KM. Percutaneous absorption of salicylic acid. Arch Dermatol. 1975;111:740-743.

44. Lindsey CP. Two cases of fatal salicylate poisoning after topical application of an antifungal solution. Med J Aust. 1968;1:353-354.

45. Fung W, Orak D, Re TA, Haughey DB. Relative bioavailability of salicylic acid following dermal application of a 30\% salicylic acid skin peel preparation. J Pharm Sci. 2008;97:1325-1328.

46. Raschke R, Arnold-Capell PA, Richeson R, et al. Refractory hypoglycemia secondary to topical salicylate intoxication. Arch Intern Med. 1991;151:591-593.

47. Goh CL, Ng SK. Contact allergy to salicylic acid. Contact Dermatitis. 1986; $14: 114$.

48. Lachapelle J-M, Leroy B. Allergic contact dermatitis to colophony included in the formulation of flexible collodion BP, the vehicle of salicylic and lactic acid wart paint. Dermatol Clin. 1990;8:143-146.

49. Kinsley M, Metelitsa AI, Somani AK. Chemical peels. In: Wolverton SE, editor. Comprehensive Dermatologic Drug Therapy. 3rd ed. New York, NY, USA: Elsevier Saunders; 2013.
Clinical, Cosmetic and Investigational Dermatology

\section{Publish your work in this journal}

Clinical, Cosmetic and Investigational Dermatology is an international, peer-reviewed, open access, online journal that focuses on the latest clinical and experimental research in all aspects of skin disease and cosmetic interventions. All areas of dermatology will be covered; contributions will be welcomed from all clinicians and

\section{Dovepress}

basic science researchers globally. This journal is indexed on CAS. The manuscript management system is completely online and includes a very quick and fair peer-review system, which is all easy to use. Visit http://www.dovepress.com/testimonials.php to read real quotes from published authors. 